\title{
A Deep Learning Method on Medical Image Dataset Predicting Early Dementia in Patients Alzheimer's Disease using Convolution Neural Network (CNN)
}

\author{
N. Deepa, S P. Chokkalingam
}

\begin{abstract}
Memory loss is one of the major dementia where the human has a common loss of memory which shows the person to behave worst and they don't care them properly. Alzheimer's disease (Ad) is a neurodegenerative disease which affects the brain with mild cognitive impairment.[4] As MCI has several phases where treatment can be consider for avoiding side effects. Deep Learning techniques is the current trend which can handle the images, massive datasets such as unsupervised, supervised and reinforcement progress.[3] A human MRI images is deal with the existing system to find the dementia. In Existing system $82.51 \%$ accuracy of classification of neural network was identified [2][3]. Due to several limitations of existing system CNN was proposed. To predict the dementia an algorithm named Logistic regression is used to produce the accuracy more than a loss function. To the test accuracy betterment OASIS project dataset is utilized.
\end{abstract}

Keywords: MCI, CNN, Alzheimer's disease, MRI, Logistic Regression, Deep Learning.

\section{INTRODUCTION}

In 1906 Dr. Alois Alzheimer was the first person who discovered the Alzheimer's disease. Dr. Alios, patient named as Auguste.D who has experienced Alzheimer's disease symptoms such as memory loss, paranoia, and psychological changes. [5] Dr. Alios noticed in the autopsy where the was in shrinkage in brain and nerve cells in the patient brain. Max Knoll and Ernst Ruska is the two persons who invented the electron microscope. The purpose of inventing that electron microscope was allowed to magnification up to 1 million times which was better magnification of normal microscope. By using this electron microscope, scientists are allowed to study the brain cells with clear understanding.[6] In 1968, Cognitive measurement scales are globally used at that period. The volume of damaged of brain tissues are estimated and measured by researchers by using this cognitive measurement scales [7][8]

\subsection{Alzheimer's Disease}

In 1974, National Institute on Aging (NIA) was established by the Congress government. From there onwards to now onwards that NIA can supports to study about this Alzheimer's disease. In 1983, November, the first National Alzheimer's disease was declared and indicating a great awareness of the disease. In 1993, the first Alzheimer's drug can approve by the Food \& Drug administration (FDA)[8]. Memory loss and dementia symptoms are the targets of this drug. From there totally five drugs are available to treat this Alzheimer's disease. In 1994, Ronald Reagan was the Former President of the United States of America has been announced the that he has been diagnosed with this Alzheimer's disease. That announcement can take a great awareness. In 2003, National Alzheimer's Disease Genetic Study (NADGS) was started by the NIA (National Institute on Aging)[9]. This NADGS gave identify the risk genes for this disease hopefully. In 2010, the sixth leading death causing disease in United States of America is the Alzheimer's disease. In 2011, the president of the United States of America was Barack Obama was signed the project named as National Alzheimer's Project Act[10][11]. This NAP act which provides to supports the national framework and fund to Alzheimer's disease. In 2013, the summit of G8 Dementia summit had launched the international effort to fight Alzheimer's and find a cure by 2025 collaborates with the United Kingdom.

Alzheimer's disease is one of the progressive brain disorder which slowly destroys memory and skills of thinking by irreversible. By WHO records, the experts suggest that the approximately 5.5 million American's who had crossed age 30 had suffered with this Alzheimer's disease. This Alzheimer's disease can also cause under age 65 [12]. Risk factor in Alzheimer's disease is the whenever the Alzheimer's disease can be treated or prevented by doctors but the problem is while in this era the population can be increased rapidly day by day. The increasing the population, spreading the Alzheimer's disease also can be increased [13]. The difference between the normal brain and the brain with $\mathrm{AD}$ as shown in the figure.
Revised Manuscript Received on October 21, 2019.

N. Deepa, Assistant Professor, Department of Computer Science and Engineering, Saveetha School of Engineering, Saveetha Institute of Medical and Technical Sciences, Chennai, India.

S P. Chokkalingam, Professor, Department of Computer Science and Engineering, Saveetha School of Engineering, Saveetha Institute of Medical and Technical Sciences, Chennai, India. 


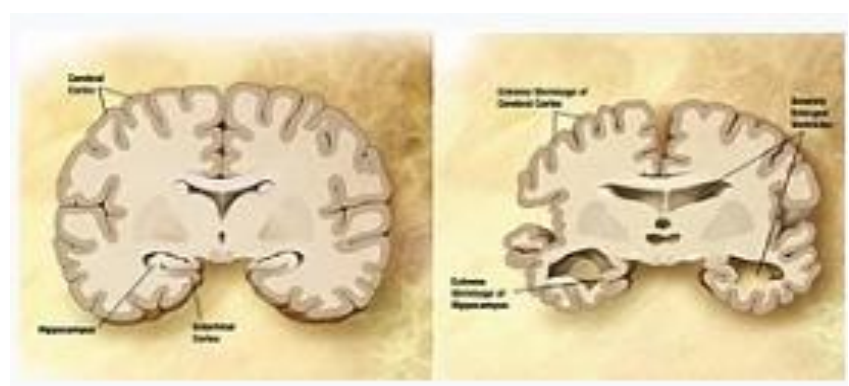

Fig. 1 Comparison of left brain and $A_{d}$ affected Brain [source : wikipedia]

Alzheimer's malady is the most widely recognized kind of dementia. Multiple brain functions and brain affected by the progressive neurological disease which is called Dementia. The major and defect of this Alzheimer's disease is the unknown, whether the number of things can be remembering but that things can be removed in a very short period of time. Alzheimer's disease (AD) is also simply referred as the Alzheimer's [14][15]. Alzheimer's is a chronic neurodegenerative disease which starts and usually worsens over a period of time. Poorly understood is the main cause of this Alzheimer's. Usually the innovation of many genes believed in the inherited from a person's parents about $70 \%$ of risk. Depression, hypertension and the head injuries are the other risk factors of this Alzheimer's.[16] The condition of Alzheimer's person is the society and family often withdraws from their family also. Ultimately the person who suffers this disease leading to death by the losing of bodily functions gradually. Neurofibrillary tangles and plaques are caused the disease associated within the brain.

\subsubsection{Symptoms and Signs}

In this Alzheimer's disease can be divided into four stages are:

They are:-

3.1) Pre-dementia stage,

3.2) Early stage,

3.3) Moderate stage and

3.4) Advanced stage.

These four stages of the disease can be divided by using the progressive pattern of functional impairment and cognitive. progress. This term of cognitive can studied about the person memory, person attention about the things and the objects and the functions which can work by the permission of the brain controls. The medical symptom term called as functional symptom which can no affect with physical body. This functional symptom can affects the nervous system of the human body. [16] And that all nervous can connected with the human brain which can be studied by the Cognitive psychology. The first stage of the Alzheimer's disease is the pre-dementia stage. In this pre-dementia stage, the first symptom is the person felling more stress than normal persons [17]. In this pre-dementia stage can be analyzing in four various type of stages where the person brain can be changed with some defects and bodily functions losing. The four stage of pre-dementia stage as given as follows:
The reading process can be involved under this cognitive

i. Prior to Diagnosis: Dementia may not be there in person's body.

ii. Stage One: Small amount of dementia may be there but the Impairment is no.

iii. Stage Two: In second stage the decline of cognitive is very less amount of mild is there.

iv. Stage Three: In third stage the decline of cognitive is increase when compare with the second stage in predementia.

This early stage is the second stage of symptoms of the Alzheimer's disease. In first stage small amount of dementia signs can't be occur whether the person can affect with the dementia but the signs can't be appear in clinical lab also [18]. But in this early stage of this disease the early stage of this dementia the person can be considered as the person who can affected by the dementia disease when take up the symptoms of the disease with clear vision. [20] This early stage of these dementia symptoms may be similar to the symptoms the person affects with 65 years old person.

This moderate stage is the of symptoms third stage of the Alzheimer's disease. This moderate stage of this disease is the person can affect with the dementia disease can occur in the medium amount. In this moderate stage the person can loss his reading and skills of writing slowly when the dementia can be increased the loss of the skills can be increased. In this moderate stage the main damaged is the person can loss his memory in a slow manner while during in this amount of period [20][21]. At the end of this moderate stage, the person can totally loss his brain memory where as the remembering the persons, remembering the objects and things and everything.

This advanced stage is the of symptoms fourth stage of the Alzheimer's disease. Advanced stage of this disease is nothing but the final stage of the Alzheimer's disease. In this advanced stage, the person can totally dependent on the caregivers. That person who has in final stage his language can completely come in simple and single words talkative and this slowly leads to the language loss. And this can leads to losing of emotional signals and the losing of verbal language with clear understand [21]. And the person with this disease can't perform even a single task with his own, he can dependent on another person (or) caregiver. Muscle mass also can be reduced slowly and comes into the small amount of the muscle mass. The Alzheimer's can't feed them because the person doesn't have muscle mass to lift her hand to the mouth and also the person forgets how to eat the food also. And this finally leads to the death of the person and the infection such as pressure ulcers also may cause to the death of the Alzheimer's.

\subsection{Causes of the Alzheimer's Disease}

The most and major cause of the Alzheimer's disease is the genetic difference between the person who can affect with this disease and unknowingly the percentage can be increased to $5 \%$ to $10 \%$.[1] The various researchers can research this phenomenon by using advance genetic technology. This causes of the Alzheimer's disease can be 
divided into the five stages are genetic, cholinergic hypothesis, amyloid hypothesis, Tau Hypothesis \& Other Hypotheses.

The genetic cause is the main problem when we come to the Alzheimer's disease. By the study of family studies and the reviews of the twins bases can explain about the disease while spreading with the genetic disorder or genetic problems. Thus studies can give the genetic diseases can ranges from $49 \%$ to $79 \%$.[2] But the studies can vary the around the $0.1 \%$ genetic causes may differ from not sex linked the persons, which can disease comes under the age of 65 years old. The main genetic cause is the autosomal. Autosomal is the one type of genetic problem which can't link with the gender between their parents. This Autosomal familial Alzheimer's disease attributed can be divided into three genes are:

They are:-

1) Amyloid Precursor Protein (APP)

3) Presenilins 2.

The genetic difference may be terminated to risk factors when the do not exhibit the autosomal inheritance. In heritance is the best known factor of the genetic disorder. APOE 4 allele is the main and most present the Alzheimer's disease person around 80\%-90\%[4]. This $\mathrm{APOE} \varepsilon 4$ allele can increase the risk factor of the disease with the increase of the three times when compare with the without this APOE\&4 allele not present in the Alzheimer's person.

Cholinergic hypothesis is the second cause of the Alzheimer's disease. This Cholinergic hypothesis is proposes the drug therapy which are currently available that proposes the Alzheimer's disease is affected by the decreasing of the synthesis name called as Neurotransmitter Acetylcholine [5]. For the purpose of the widespread support this cholinergic hypothesis is not maintained for a long period of time.

Amyloid Hypothesis is the third cause of the Alzheimer's disease. The first era of this amyloid hypothesis at 1991. In 1991 , the researchers can founded the amyloid hypothesis is the one of the fundamental cause of the Alzheimer's disease. Chromosome 21 is the template of this hypothesis which can be identified by the Amyloid Precursor Protein (APP). When the doctors (or) the researchers can be breakdown this amyloid hypothesis at the time also the disease may not be cure because of the some risk factors are present name called as APOE4. For this problem the researchers can found the vaccine which works on the human trials experimentally but not the dementia effecting of the Alzheimer's disease [6]. For this amyloid hypothesis, theory can be updated at 2001 which can be works on the basics of the protein name called as the beta-amyloid protein. By using this theory mechanism can be discovered name called as the amyloid-related mechanism. This amyloid-related mechanism can be connections in the brain between the prunes neuronal. These prunes neuronal can be using purpose of the fast-growth phase of early-life. The drug name called as the DR6 is the main cause affected the Alzheimer's disease which can highly express in the brain. For depressing synaptic function, this beta-amyloid protein
2) Presenilins $1 \&$

can plays a major role while treating of this particular cause hypothesis [8].

This Tau Hypothesis is the fourth cause of the Alzheimer's disease. The disease cascade initiates by the abnormalities of the Tau Protein which is invented by this Tau Hypothesis. Nerve cells which are presented in the inside of the human body can eventually, forming their tangles called neurofibrillary tangles. When this Neurofibrillary tangles can occur automatically the disintegrate of the microtubules are started. Neuron transport system can be collapsed by the destroying the cell's cytoskeleton of the structure[11]. This damage can finally occur to the malfunctions between the neurons and biochemical communication. And the patient cells lead to the death cells. The tau protein can break the brain cells as shown in the figure.

This other hypothesis is the last cause of the Alzheimer's disease. When the Blood brain-barrier may be involved in the poor functioning state were the proposed can b e arise in the neurovascular hypothesis. In this neurovascular hypothesis, the dementia had been linked with the functions. The name of the functions called as Spirochete infections.

\section{CNN TECHNIQUE}

CNN stands for convolutional neural network algorithm. $\mathrm{CNN}$ is also known as the ConvNet where it can be a classical deep neural network [19]. This deep neural network is most probably used for the analyzing of visual memory. Multilayer perceptron's is the regularized versions in the CNN. The fully networks are interconnected by referring this multilayer perceptron's. When the networks are interconnected, each and every neuron interconnected with the layer of the neuron. When we go through the regulation process this $\mathrm{CNN}$ can give a different approaches while compare with the other neural network algorithms. CNNs are the lower extreme when we compare with the complexity and the connectedness on the scale. The shift variant may be able to be differing in the shared-weights architecture.[19] This variant differ is known as the Shift Invariant (or) space invariant artificial neural networks (SIANN). By using the mathematical operation the network employs indicating the convolutional neural networks called as Convolution. Linear operation is a special king in the convolution. Convolution neural networks are also known as the simple neural networks which can be utilized for the purpose of the general matrix multiplication of one layer at least.

\subsection{Image Recognition with Cnns Trained Data:-}

In 1989 this image recognition with CNNs trained by gradient descent can be introduce by the Yann LeCun. By using this image recognition with CNNs trained by gradient the system can be utilized by the ZIP files and drag the image. CNNs can be designed following by the five stages are:

1. Target Image,

2. Feature Maps, 
3. Rectified Feature Maps

5. Output.

4. Fully Rounded Triangle \&

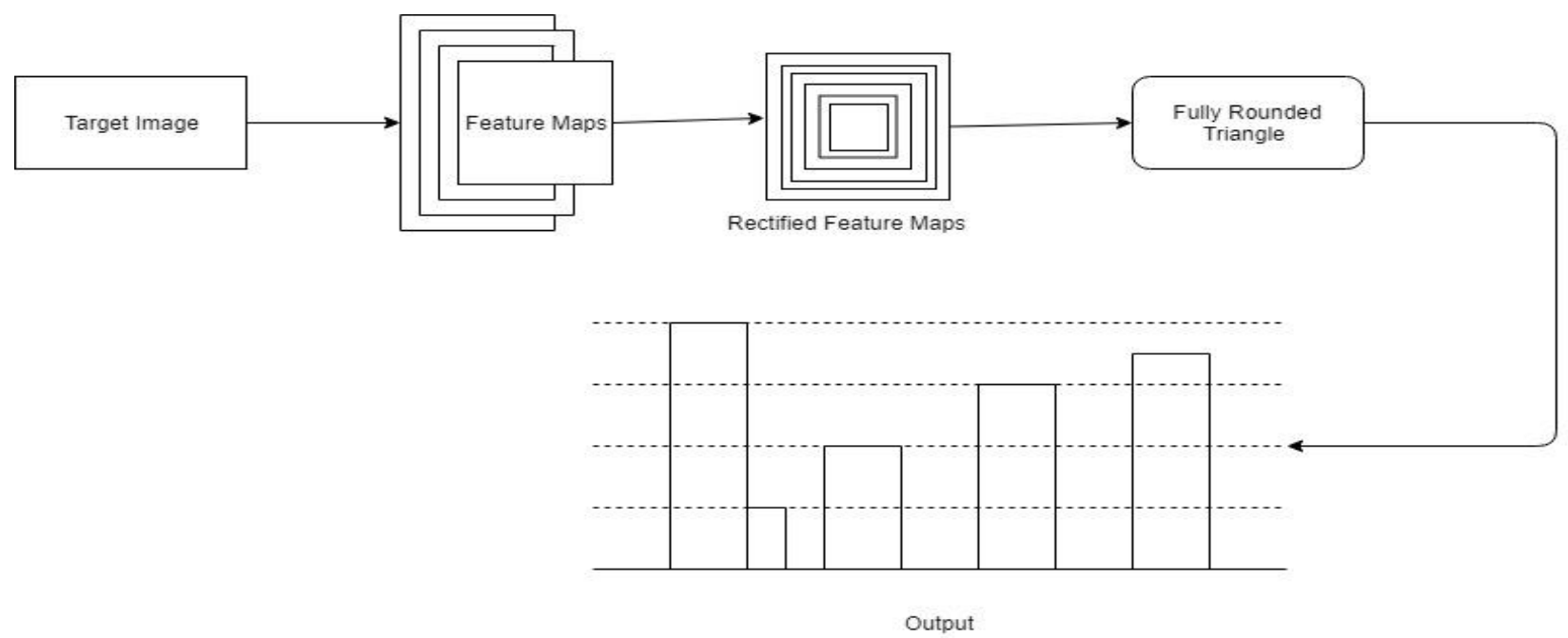

Fig. 2 CNN flow diagram

Step-1:- Input can be taken as the Target Image.

Step-2:- After the giving the inputs the Image can be go to the Feature Maps.

Step-3:- That the Feature maps can be converted into the Rectified Feature Maps.

Step-4:- After converting the rectified feature maps, the image can go through the fully rounded Triangle.

Step-5:- And the finally the output can be displayed.

\subsection{Existing System}

The main involvement of this literature filed is focus on the Alzheimer's disease effects on the people who suffer with genetic and health issues in brain. The Alzheimer's had the impaired available of vitamins, proteins and plaques in the brain[16]. When we go through this literature the main strength of this field is how the disease can attack the person and how the disease can be spread person to person with genetic problem in their genes. And we can understand what the causes of the Alzheimer's disease are and this field tells about the prevention of this disease [11]. The peoples can identify the Alzheimer's by verifying the signs and symptoms in the field. The major drawback is the how to cure this Alzheimer's disease. Because the still research can go through the cure of this disease. The NIA still works on it.

\subsection{Proposed System}

Before all existing system focus on the prediction of the Alzheimer's disease by using MRI imaging reports of the patient and predicted the disease. But the problem behind the existing system they didn't focus on the accuracy of the disease prediction. In this proposed system we can increase the test accuracy of the Alzheimer's disease and increase the efficiency of the result of the testing reports. For this increasing the accuracy of the testing disease we apply the logistic regression algorithm to the dataset while executing the test case.

Logistic Regression Algorithm is used for the predict analysis of the dataset with the high rate accuracy of the resulted output of the dataset. The working principle of this logistic regression algorithm, it can be divided into classifications tasks while we can train the dataset from into the program. Logistic regression is the remodel of the linear regression for the purpose of the increasing the rate of accuracy. Sigmoid Function can be used in this Logistic Regression function type of the calculation.

MRI stands for Magnetic Resonance Imaging. This Magnetic Resonance Imaging is nothing but the imaging technique used for the medical reports in labs[6]. The Magnetic Resonance Imaging used in the Radiology laboratories for the forming of the anatomy pictures. And it is also used in the Physiological process of the human body. In magnetic resonance imaging process having the huge and strong strength of the magnetic fields gradients and strong magnetic fields [12]. In MRI scanners to purpose of the generation of the parts or organs of the human body by using of the radio waves.

\section{IMPLEMENTATION}

Implementation part can be done in the three major sub divisions of the project.

They are:-

1) Dataset and

2) Train The Data.

\subsection{Dataset}

This Dataset consists of the OASIS project data which is based on the MRI imaging scanning images of the Alzheimer's disease. The dataset can be divided into two dataset types are there:

a) Cross-sectional MRI Data and

b) Longitudinal MRI Data.

\section{Cross-sectional MRI Data \& Longitudinal MRI Data.}

This cross-sectional MRI data consists of the young aged, middle aged, Non-demented and Demented Older Adults. And this set of cross-sectional MRI data consists of the collection of the 416 subjects from aged 18-96. In each 
subject, TI individuals of 3 or 4 branches is there in the dataset. This Longitudinal MRI data consists of the Nondemented and Demented Older Adults [14]. And this set of
Longitudinal MRI data consists of the collection of the150 subjects from aged 60-96. In each subject, TI individuals of 3 or 4 branches is there in the dataset.

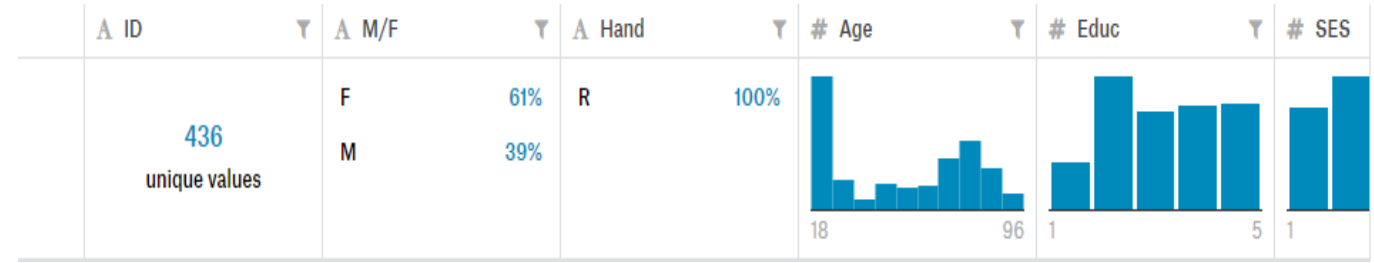

Fig 3.1.1 Cross-sectional MRI Dataset

\begin{tabular}{|c|c|c|c|c|c|c|c|c|c|c|}
\hline A Subject ID & $T$ & A MRIID & $T$ & A Group & $\mathbf{T}$ & \# Visit & $\mathbf{T}$ & \# MR Delay & $\mathbf{T}$ & A $\mathrm{M} / \mathrm{F}$ \\
\hline OAS2_0048 & $1 \%$ & & & Nondemented & $51 \%$ & & & & & $\mathrm{~F}$ \\
\hline OAS2_0070 & $1 \%$ & $\begin{array}{c}373 \\
\text { unique values }\end{array}$ & & Demented & $39 \%$ & & & & & M \\
\hline Other (148) & $97 \%$ & & & Other (1) & $10 \%$ & 1 & 5 & 0 & 639 & \\
\hline
\end{tabular}

Fig 3.1.2 Longitudinal MRI dataset

\subsection{Train the Data}

For the training the data we use the logistic regression algorithm and by using the python platform for using more libraries at a time and in calculation part of the logistic regression algorithm we can use the sigmoid function for get more efficiency by divided and combining of performing the classification tasks while executing time

\subsection{Result}

\section{- Data View}

The data view is nothing but the import the data from the dataset of the cross-sectional MRI data and Longitudinal MRI data.

\section{oasis_cross-sectional.csv \\ oasis_longitudinal.csv}

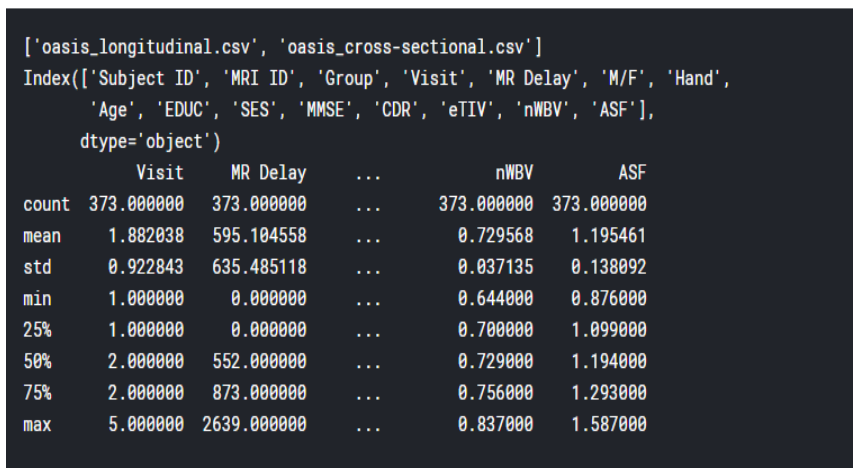

Fig 3.3.1 Data View

\section{- Viewing by Rows and Columns}

The viewing by rows and columns is nothing but the arrange the data in a row wise and column wise.

\section{- Calculating the Leaf nodes}

Leaf nodes are nothing but the calculating the max leaf nodes and the mean absolute error values in a arranging of the row and column wise.

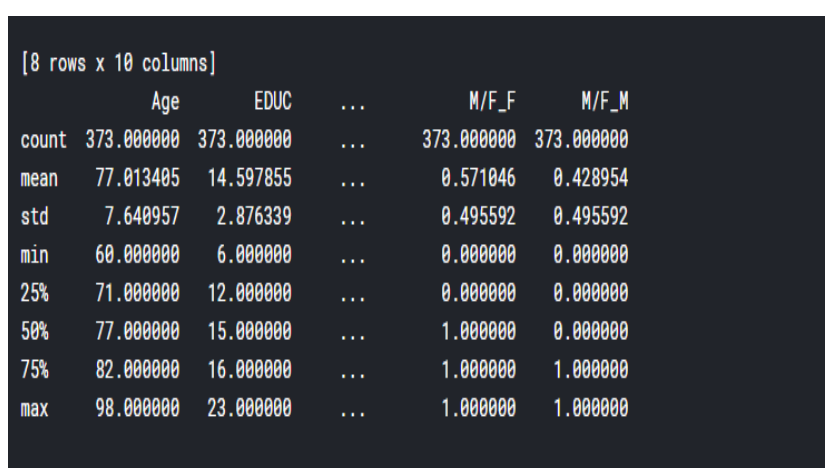

Fig 3.3.2 Rowwise and columnwise Data

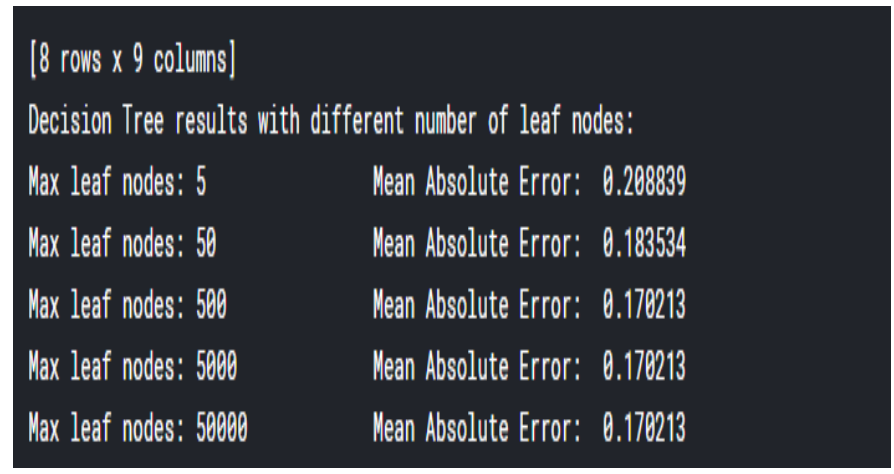

Fig 3.3.3 Max and Mean of Leaf node

\section{- Arranging the dataset columns}

Arranging the dataset columns is the process of the creating new data frames to the coding by using the pandas library function.

\section{- Obtain the variable data}

Obtain the variable data is the process of the taking the data from the data frames and present in the consisting of their particular declaration of the variable.

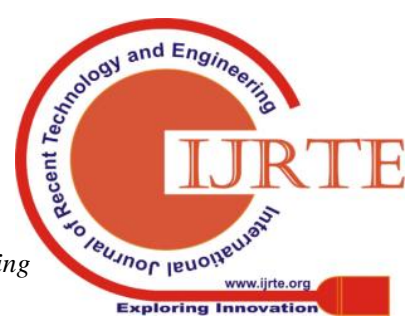




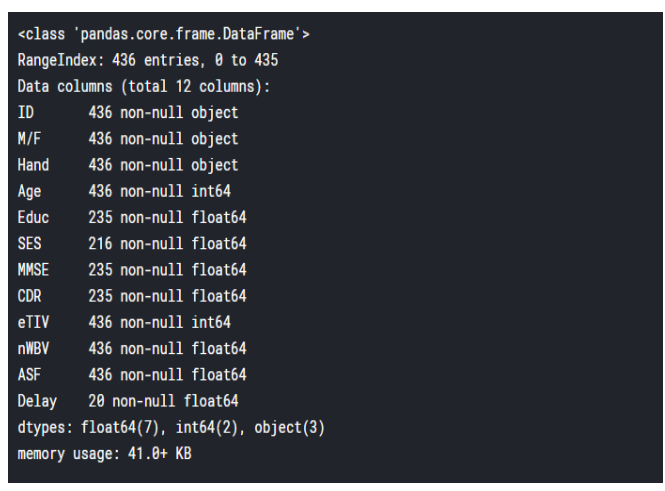

Fig 3.3.4 Pandas Library packages

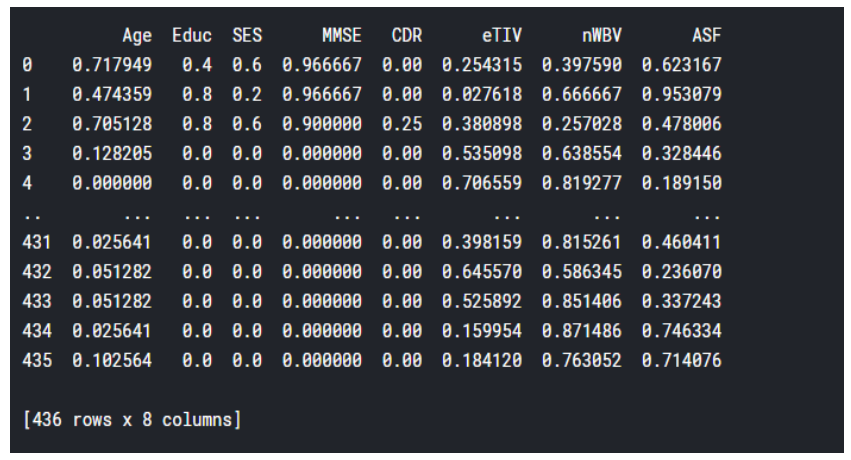

Fig 3.3.5 locating the data

\section{- Test Accuracy result}

Accuracy of the result disease is shown in fig.

\section{testaccuracy: 90.56537}

Fig 3.3.6 Result Accuracy

\section{CONCLUSION}

In this information age technology can be improved in very fast growth as well as well disease in the world can be spread very fast [9]. For this condition we can use that same technology in a proper manner and predicted the diseases easily and cure the diseases very fast. The before all existing can predicted the disease but this proposed system we can predicted the disease and increasing the test accuracy of the disease and giving more efficient information about the patient reports of the MRI imaging scanners by comparing the old patient disease who have the already suffered that disease [14]. For making the better results we can added the clear vision of the disease such as history of the disease, detailed causes of the disease, identification of signs and symptoms, about the pathophysiology and ways to prevention of this disease [17]. The future work of this research can concentrate on the resolve that spreading ways of issues and the ways to identify the curing ways by study of the some research.

\section{REFERENCES}

1. L. Wang et al. (Eds)(2016), ' Deep Ensemble Sparse Regression Network for Alzheimer's Disease Diagnosis', Springer International Publishing AG 2016, MLMI 2016LNCS 10019,pp. 113-121, 2016.

2. https://www.kaggle.com/hyunseokc/detecting-early-alzheimer-s\#6. MODEL.
3. Debesh Jha and Goo-Rak K won, Chosun University, Gwangju, Republic of South korea, 'Diagnosis of ALZHEIMER'S DISEASE Using A Machine learning Technique', Poster Presentations, refernce no.P1538.

4. https://www.alz.org/alzheimers-dementia/what-is-dementia

5. Clifford R. Jack, Jr, Marilyn Albert, David S. Knopman, Guy M. McKhann, Reisa A. Sperling, Maria Carillo, William Thies, and Creighton H. Phelps,'Introduction to Revised Criteria for the Diagnosis of Alzheimer's Disease: National Institute on Aging and the Alzheimer Association Workgroups', from the National Institute on AgingAlzheimer's Association workgroups on diagnostic guidelines for Alzheimer's disease. Alzheimers Dement 2011;7(3): 257-262.

6. Martijn C. deWildea, Bruno Vellasb, Elodie Giraulta, Aysun Cetinyurek Yavuza, JohnW. Sijben, (Eds)(2017), 'Lower brain and blood nutrient status in Alzheimer's disease: Results', Alzheimer's \& Dementia: Translational Research \& Clinical Interventions 3 (2017) 416-431.

7. Guoqiao Wanga,*, Chengjie Xionga, Eric M. McDadeb, Jason Hassenstabb, Andrew J. Aschenbrennerb, Anne M. Faganb, Tammie L. S. Benzingerc, Brian A. Gordonc, John C. Morrisb, Yan Lib, Randall J. Batemanb, the Dominantly Inherited Alzheimer Network(DIAN), 'Simultaneously evaluating the effect of baseline levels and longitudinal changes in disease biomarkers on cognition in dominantly inherited Alzheimer's disease, Alzheimer's \& Dementia: Translational Research \& Clinical Interventions 4 (2018) 669-676.

8. David G. Munoz, Howard Feldman, 'Causes of Alzheimer's disease', CMAJ 2000;162(1):65-72.

9. Hongming Lia, Mohamad Habesa,b, David A. Wolkb, Yong Fana, 'A deep learning model for early prediction of Alzheimer's disease dementia based on hippocampal magnetic resonance imaging data', Alzheimer's \& Dementia - (2019) 1-12.

10. David V. Hansen, Jesse E. Hanson, and Morgan Sheng, 'Microglia in Alzheimer's disease', Published Online: 1 December, 2017 | Supp Info: http://doi.org/10.1083/jcb.201709069.

11. Vanessa J De Paula, 'Alzheimer's disease', Article in Sub-cellular biochemistry · December 2012,DOI: 10.1007/978-94-007-5416-4_14.

12. Alistair Burns, Steve Iliffe, 'Clinical Review of Alzheimer's disease', Cite this as: BMJ 2009;338:b158,doi:10.1136/bmj.b158.

13. Yiming Ding, 'A Deep Learning Model to Predict a Diagnosis of Alzheimer Disease by Using 18F-FDG PET of the Brain', Radiology 2018; 00:1-9 • https://doi.org/10.1148/radiol.2018180958.

14. Dennis J Selkoe1, \& John Hardy2, 'The amyloid hypothesis of Alzheimer's disease at 25 years', EMBO Molecular Medicine Vol 8 No $6 \mid 2016$.

15. Igor O. Korolev, 'Alzheimer's Disease: A Clinical and Basic Science Review', College of Osteopathic Medicine and Neuroscience Program, Michigan State University, East Lansing, MI, USA.

16. Ammarah Farooq, Syed Muhammad Anwar, Muhammad Awais, Saad Rehman, 'A deep CNN based multi-classification of Alzheimer's Disease using MRI.

17. Eric J. Lien, James D. Adams, Linda L. Lien and Meng Law, 'Alternative Approaches to the Search for Alzheimer's Disease Treatments', Department of Pharmacology and Pharmaceutical Sciences, School of Pharmacy, University of Southern California, Los Angeles, CA 90899-9121, USA; elien@usc.edu.

18. Debesh Jha and Goo-Rak Kwon, Chosun University, Gwangju, 'Diagnosis of Alzheimer's Disease Using a Machine Learning Technique', Republic of South Korea. Contact e-mail: grkwon@chosun.ac.kr.

19. N.Deepa, SP.Chokkalingam, "Deep Convolutional Neural Network (CNN) for Medical Image Analysis", International Journal of Engineering and Advanced Technology (IJEAT) ISSN: 2249 -8958, Volume-8, Issue-3S, February 2019.

20. Donghuan Lu, Karteek Popuri, Gavin Weiguang Ding, Rakesh Balachandar, Mirza Faisal Beg, 'Multiscale Deep Neural Networks based analysis of FDG-PET images for the Early Diagnosis of Alzheimeros Disease', Medical Image Analysis (2018), doi: 10.1016/j.media.2018.02.002.

21. Mendiola-Précoma, Rodríguez-Cruz A1, Berumen and García-Alcocer, 'The Etiology of Alzheimer's Disease', García-Alcocer G, Laboratorio de Investigación Genética, Universidad Autónoma de Querétaro, México. 